\title{
Commensurability, excitation gap and topology in quantum many-particle systems on a periodic lattice
}

\author{
Masaki Oshikawa \\ Department of Physics, Tokyo Institute of Technology, Oh-okayama, Meguro-ku, Tokyo 152-8551, Japan
}

(August 9, 1999)

\begin{abstract}
Combined with Laughlin's argument on the quantized Hall conductivity, Lieb-Schultz-Mattis argument is extended to quantum many-particle systems (including quantum spin systems) with a conserved particle number, on a periodic lattice in arbitrary dimensions. Regardless of dimensionality, interaction strength and particle statistics (bose/fermi), a finite excitation gap is possible only when the particle number per unit cell of the groundstate is an integer.

PACS numbers: 71.10.Fd,75.10.Jm,75.60.Ej
\end{abstract}

Strongly interacting quantum many-particle systems is one of the central topics of theoretical physics. The Renormalization Group (RG) is an important concept in studying such problems [1]. According to the RG picture, low-energy, long-distance behavior of a quantum many-particle system is governed by RG fixed points. A quantum critical system, which has gapless excitation spectrum, is renormalized into a critical RG fixed point. However, in general, a critical RG fixed point allows some relevant perturbations. When the perturbations are added, the system is driven away from the critical fixed point. Such system generally has a finite excitation gap. Then it is expected that a gapless quantum critical system is unstable and only achieved by an appropriate fine-tuning of the Hamiltonian, which makes the relevant perturbations vanish.

In reality, there are rather many quantum critical system with gapless excitation spectrum, obtained without any apparent fine-tuning. Thus, one may naturally ask a question: is there some mechanism which protects a gapless critical system? There is one well-known mechanism of such kind: a gapless Nambu-Goldstone mode [2] exists when the system has a spontaneously broken continuous symmetry. In fact, it describes variety of gapless excitations in quantum many-body systems, like spin waves and phonons, etc. However, it does not exhaust all the (stable) quantum critical systems. On the other hand, few universal mechanism other than the NambuGoldstone theorem are known [3].

In this letter, we argue that an incommensurability is another universal mechanism which protects the gapless excitation spectrum in quantum many-particle systems. We will show the following statement:

In a quantum many-particle system defined on a periodic lattice, with an exactly conserved particle number, a finite excitation gap is possible only if the particle number per unit cell of the ground state is an integer.

This condition to have a finite gap may be called as the "commensurability condition". When the particle number per unit cell [4] of the lattice is $\nu=p / q$ where $p$ and $q$ are coprimes, a gapful groundstate must spontaneously break the translation symmetry, so that the unit cell of the groundstate is enlarged by factor of $q$. In the case of quantum spin system with the conserved total magnetization $\sum_{j} S_{j}^{z}$, mapping of the spin system to an interacting boson system gives $S-m$ particles per spin where $S$ is the spin quantum number, $m$ is the average magnetization per spin.

An incommensurate filling corresponds to the limit of large $q$, giving large ground state degeneracy if there is a finite excitation gap. This usually implies the spectrum is actually gapless. That the incommensurate filling gives a gapless spectrum is empirically recognized more or less. In fact, all trivial ground states (e.g. completely dimerized state) with an excitation gap, as well as the less trivial Valence-Bond-Solid states [5], satisfy the commensurability condition. However, it is not trivial whether the incommensurability generally guarantees gapless excitations in the presence of interaction and quantum fluctuation.

If a gapful phase is adiabatically connected to those trivial states within the Hamiltonians conserving the particle number and periodicity, it must also satisfy the same commensurability condition. This is because an infinitesimal modification of the Hamiltonian does not mix states with different particle number; thus the particle number per unit cell in the groundstate is unchanged during the adiabatic change, if the gap always remains open. This explains the stability of the particle density $\nu$ in such cases (e.g. in the "strong coupling" approach to the magnetization plateau [6]). However, this argument does not exclude the possibility of a gapful phase, which is not adiabatically connected to any trivial state, not obeying the commensurability condition.

In this letter, based on a topological argument, we will show that the commensurability condition is a robust non-perturbative constraint. We consider general quantum many particle systems on a lattice with a periodic structure with any strength of interaction, in $D$ dimensions. For simplicity, here we assume that there is a single species of particles. We assume that the number of particles is conserved (i.e. commutes with the Hamil- 
tonian.) Let us call the direction of an arbitrary chosen primitive vector $\vec{a}$ of the lattice as $x$-direction. We impose the periodic boundary condition in $x$-direction with length $L$ measured in unit of $|\vec{a}|$. Defining the translation operator $T_{x}$ which translates the system by $\vec{a}$, the periodic boundary condition is represented as $T_{x}{ }^{L}=1$, and the Hamiltonian invariant under the translation $T_{x}$. The "cross section" of the lattice is defined so that the whole lattice is spanned (without overlap) by translation of the cross section by $T_{x}$. We denote the number of unit cells contained in the cross section by $C$; the total volume (ie. number of unit cells) of the system is given by $C L$.

In one dimension (including ladders etc.), the proposed statement was already shown in Refs. [7]:8] by generalizing the Lieb-Schultz-Mattis (LSM) argument [9]. Therefore the remaining problem is to understand higher dimensions. Applying the LSM argument to the higher dimensions $D>1$ meets a difficulty. The energy of the variational state is bounded only by $O(C / L)$, which is generally not small in the thermodynamic limit; in an isotropic (in size) system, $C \sim L^{D-1}$.

Affleck 10 discussed some application of the LSM argument to $D>1$. While in Ref. [10] only spin systems at zero magnetization were considered, it is straightforward to extend the discussion in Ref. [10] to quantum manyparticle systems with general particle density (quantum spin systems with general magnetization), as was done in one dimension [7, 8]. Unfortunately, the strong anisotropy limit $C / L \rightarrow 0$ is necessary to apply the LSM argument as in Ref. 10]. He argued it plausible that the conclusion is still valid for an isotropic system where $C \sim L^{D-1}$. However, the strong anisotropy limit makes the system essentially one-dimensional. Thus one might suspect that the LSM argument does not give a useful information on higher-dimensional system which is isotropic in size. Below we will argue that the same conclusion holds, without relying on the strong anisotropy limit.

As in Ref. [10], we impose the periodic boundary condition for $x$-direction, and require $C$ to be mutually prime with $q$ but not the anisotropy condition $C \ll L$. The boundary conditions for other than $x$-direction can be either open or periodic if they are uniform in $x$-direction. The particles may or may not have a real electric charge. Here we introduce a fictitious charge $e$ for each particle, which couples to an externally given (fictitious) electromagnetic field.

Because of the periodic boundary condition in the $x$ direction, the system may be regarded as a ring. Following Laughlin's discussion [11] of the Quantum Hall Effect (QHE), we consider a magnetic flux $\Phi$ piercing through the ring. In a simplest gauge choice. the magnetic flux can be represented by the uniform vector potential $A_{x}=\Phi / L$ in the $x$-direction, Now let us adiabatically increase the magnetic flux $\Phi$ by a unit flux quantum $\Phi_{0}=h c / e$, when the system is in the groundstate $\left|\Psi_{0}\right\rangle$ at $\Phi=0$. The groundstate $\left|\Psi_{0}\right\rangle$ is chosen (when the groundstates are degenerate) so that it is also an eigenstate of $T_{x}$. This is always possible, at least in a finite size system, because we assumed periodic lattice structure and periodic boundary condition in $x$-direction; the Hamiltonian commutes with $T_{x}=e^{i P_{x}}$. Here $P_{x}$ is the $x$-component of the total (crystal) momentum. The groundstate is thus also an eigenstate of the momentum with an eigenvalue $P_{x}^{0}$ :

$$
P_{x}\left|\Psi_{0}\right\rangle=P_{x}^{0}\left|\Psi_{0}\right\rangle,
$$

During the adiabatic process, the Hamiltonian is only modified by the uniform vector potential $A_{x}=\Phi / L$ in the above gauge choice. Then the Hamiltonian always commutes with $T_{x}$. When the magnetic flux reaches the unit flux quantum, the original groundstate evolves into some state $\left|\Psi_{0}^{\prime}\right\rangle$. The Hamiltonian $H(\Phi)$ generally depends on the flux $\Phi$ through the vector potential, reflecting the Aharanov-Bohm (AB) effect. However, when the $\mathrm{AB}$ flux $\Phi$ is equal to the unit flux quantum, there is no $\mathrm{AB}$ effect and the energy spectrum is identical to the zero flux case. In fact, the vector potential is eliminated by the large gauge transformation

$$
U=\exp \left[\frac{2 \pi i}{L} \sum_{\vec{r}} x n_{\vec{r}}\right]
$$

where $n_{\vec{r}}$ is the particle number operator at site $\vec{r}$, and $x$ is the $x$-coordinate of $\vec{r}$. Namely, the Hamiltonian with the unit flux quantum goes back to the original one by the large gauge transformation as $U H\left(\Phi_{0}\right) U^{-1}=H(0)$. By the same transformation, the adiabatic evolution of the groundstate $\left|\Psi_{0}^{\prime}\right\rangle$ is mapped to $U\left|\Psi_{0}^{\prime}\right\rangle$. Thus, after the adiabatic procedure and the large gauge transformation, we get back to the original Hamiltonian but the groundstate $\left|\Psi_{0}\right\rangle$ is changed to $U\left|\Psi_{0}^{\prime}\right\rangle$.

On the other hand, in the presence of a finite excitation gap, the ground state $\left|\Psi_{0}\right\rangle$ can only be transformed into itself or, possibly, into another one of degenerate groundstates during the adiabatic process [11, 12]. As already explained, the reason why the LSM argument has been applied only to one dimension (or to the strong anisotropic limit) is that, the energy expectation value of the variational state is bounded only by $O(C / L)$, which is generally not small. Thus one can not say $U\left|\Psi_{0}\right\rangle$ is always a low-energy state in $D>1$ dimensions. However, applying the adiabatic argument, we are able to claim that, if the system has a finite excitation gap, the outcome of the adiabatic evolution $U\left|\Psi_{0}^{\prime}\right\rangle$ should be one of the groundstates 14 .

In the case of QHE, an implicit assumption 11] of uniqueness of the groundstate led to an integer quantization of Hall conductivity. However, as pointed out by Tao and $\mathrm{Wu}[12]$, it is possible that the ground states are degenerate, and that is what needed in fractional QHE. Therefore we have to check whether $U\left|\Psi_{0}^{\prime}\right\rangle$ is identical to $\left|\Psi_{0}\right\rangle$ or not. 
Here let us recall that, $U\left|\Psi_{0}\right\rangle$, which is similar to $U\left|\Psi_{0}^{\prime}\right\rangle$, is nothing but the variational state constructed in the LSM argument and its generalizations [9, 13, 10, 7]. Now we see a rather close relation between LSM and Laughlin's arguments. While our state $U\left|\Psi_{0}^{\prime}\right\rangle$ is not identical to the LSM state $U\left|\Psi_{0}\right\rangle$, we can still invoke the LSM orthogonality argument used for $U\left|\Psi_{0}\right\rangle$.

We have chosen the original groundstate as an eigenstate of $P_{x}$ as in eq. (11). Since the Hamiltonian always commutes with $P_{x}$ during the adiabatic process, the eigenvalue of $P_{x}$ is unchanged. This can be seen easily from perturbation theory on an infinitesimal increase of the $\mathrm{AB}$ flux; the infinitesimal modification of the Hamiltonian commutes with $P_{x}$ and it does not mix states with different eigenvalues of $P_{x}$. Thus, $\left|\Psi_{0}^{\prime}\right\rangle$ belongs to the same eigenvalue $P_{x}^{0}$ as in eq. (1). Now, after the gauge transformation, $\left|\Psi_{0}^{\prime}\right\rangle$ is transformed to $U\left|\Psi_{0}^{\prime}\right\rangle$, which may belong to a different eigenvalue. By using the identity

$$
U^{-1} T_{x} U=T_{x} \exp \left[2 \pi i \sum_{\vec{r}} \frac{n_{\vec{r}}}{L}\right]
$$

we see that $U\left|\Psi_{0}^{\prime}\right\rangle$ is an eigenstate of $P_{x}$ with $P_{x}=$ $P_{x}^{0}+2 \pi \nu C$. Thus, if choose $C$ to be mutually prime with $q(\nu=p / q), U\left|\Psi_{0}^{\prime}\right\rangle$ is orthogonal to $\left|\Psi_{0}^{\prime}\right\rangle$ and $\left|\Psi_{0}\right\rangle$, because it belongs to a different eigenvalue of $P_{x}$. By repeating the same procedure several times, we can conclude that there are at least $q$ degenerate ground states. Thus we have shown that the similar conclusion to the one-dimensional case 0 , 8 holds in any dimensions, without relying on the strong anisotropy limit. The optimistic view taken in Ref. 110] is actually justified, as far as the anisotropy condition is concerned. It is valid for arbitrary strong interaction, and is even independent of the particle statistics (bose/fermi). The ground state degeneracy is a robust, non-perturbative property related to the topology of the gauge field and the symmetries of the system.

An unsatisfactory point still remaining is that we have to take $C$ to be mutually prime with $q$. If $C$ is an integral multiple of $q$, nothing can be said on the degeneracy, and a unique ground state with an excitation gap is possible in principle. This is also related to the fact that the present argument is not yet mathematically rigorous for the thermodynamic limit. (Compare to Ref. [13].) However, if we assume that the groundstate degeneracy does depend on whether $C$ is an integral multiple of $q$ or not in a large enough system, it suggests some long-range structure of period $q$. Then it is naturally expected [10] that the ground states have the $q$-fold degeneracy anyway, for a sufficiently large system. In addition, the present argument can be applied to many different boundary conditions, because there are various possible choices of the primitive vector $\vec{a}$ and the corresponding cross section. The degeneracy looks less artificial in the light of this fact.
The ground states in a finite system would be actually split by exponentially small energy due to tunneling effects. In a finite size system, the $q$ (near-)groundstates $\left|\Psi_{n}\right\rangle(n=0,1, \ldots, q-1)$ are eigenstates of the momentum $P_{x}: P_{x}\left|\Psi_{n}\right\rangle=(2 \pi n / q)\left|\Psi_{n}\right\rangle$. However, in the thermodynamic limit, the physical groundstates are given by $\left|\tilde{\Psi}_{j}\right\rangle$ 's, which are defined as $\left|\tilde{\Psi}_{j}\right\rangle=\sum_{n} e^{i P_{x} j}\left|\Psi_{n}\right\rangle$. These physical ground states are connected by the translation operator:

$$
T_{x}\left|\tilde{\Psi}_{j}\right\rangle=\left|\tilde{\Psi}_{(j+1) \bmod n}\right\rangle .
$$

Thus the translation symmetry (to $x$-direction) is spontaneously broken, and the periodicity of the ground state is an integral multiple of $q$. This concludes the derivation of the proposed statement.

We note that, Lee and Shankar 15] had derived a similar statement for the limited case of hard-core models in two dimensions. However, their argument relies on a certain field-theory mapping, and looks less reliable compared to ours. In fact, their statement appears to be too strong: they state that there must be a Charge Density Wave (CDW) order if the system with a fractional filling $\nu<1$ has a gap. This has a rather simple counterexample: the spontaneously dimerized ground state of a $S=1 / 2$ Heisenberg magnet at zero magnetization, which corresponds to a hard-core boson system with $\nu=1 / 2$, has no long-range Néel-type (namely, CDW) order. On the other hand, the spontaneously dimerized state does break the translational symmetry, and is consistent with our conclusion.

The ground state degeneracy of $S=1 / 2$ quantum spin systems has been discussed in several different contexts, for example in Ref. [16]. In particular, there has been a lot of discussions on the possibility of the exotic spinliquid state called the Resonating Valence Bond (RVB) state. While various possibilities were considered under the name of RVB, here we refer to the proposals [17, 18 of a disordered groundstate with a finite excitation gap, but without any apparent breaking of the translation symmetry. If there is really such a spin-liquid state, it appears contradictory to our result. This might be the reason [10] why there is no established example of such an RVB groundstate, despite intensive search in various $S=1 / 2$ spin systems with odd number of spins per unit cell.

However, in spite of its uniform appearance, some degeneracy is argued to exist 17,19] in the RVB state, under the periodic boundary condition. In Refs. 117, 19, this degeneracy is argued to be unphysical. For the degeneracy to be unphysical, it must be that no physical operators distinguish the groundstates with the spontaneously broken translation symmetry. While we are not sure it is possible, we do not rule out such a possibility. In any case, the (near-) degeneracy of the finite size system concluded from the present argument seems consistent with Refs. 17, 19]. 
The ground-state degeneracy in a gapped phase required by the present argument, is related to the commensurability. Intuitively, this is quite natural; the particles can be locked into a stable groundstate only when it can have a commensurate structure with the lattice. Such an intuition is, however, more or less based on some trivial states which can be easily imagined in minds. Nevertheless, the commensurability condition turned out to be essential even in the presence of arbitrarily strong interaction and quantum fluctuation, because of the topological mechanism discussed in the present letter.

In generic cases, a finite excitation gap would be only possible at special commensurate (rational) filling. In case of charged particles, such a gapped phase includes Mott and band insulators. On the other hand, gapless phases at generic filling includes superfluid, Fermi liquid and possibly other conducting phases. In case of quantum spin systems, the gapful phases are related to the magnetization plateau at quantized magnetization. In any dimensions, a magnetization plateau with a finite excitation gap is possible only if the commensurability condition is satisfied: $n(S-m)=$ integer where $n$ is the number of spins in the unit cell of the ground state, similarly to the one-dimensional case [7]. In fact, several magnetization plateaus reported in $D>1$ dimensions (examples include 20]) satisfies this quantization condition. However, a plateau in a magnetization curve appears if there is no gapless excitation which changes the total magnetization. As already mentioned in Ref. [7], the LSM argument (and the present argument) does not directly guarantee gapless excitations of such kind. Thus, it may be possible to have an "exceptional" magnetization plateau which does not obey the above simple quantization condition, if there are gapless excitations only with the same total magnetization as the groundstate. In one dimension, all plateaus should obey the quantization condition as far as the general Abelian bosonization treatment [7] is valid. (However, see 21] for a possible exceptional "plateau" at $m=0$, and [22 for a discussion in the doped case.) In higher dimensions, the situation is less clear, while certainly many plateaus 20 satisfy the simple quantization condition. An exceptional plateau at $m=0$ might be realized in Kagomé lattice [23], which is argued to have singlet gapless excitations.

Finally, we note that our commensurability condition has obvious generalizations to the spinful electron systems, Kondo lattices, and other multi-species particle systems in arbitrary dimensions.

I would like to thank Ian Affleck, Hal Tasaki and Masanori Yamanaka for many stimulating discussions over several years. I also thank Subir Sachdev for pointing out some relevant references. This work is supported by a Grant-in-Aid from Ministry of Education, Science, Sports and Culture of Japan.
[1] K. G. Wilson and J. Kogut, Phys. Rep. 12C, 75 (1974).

[2] Y. Nambu, Phys. Rev. Lett. 4, 380 (1960); J. Goldstone, Nuovo Cimento, 19, 154 (1961).

[3] I. Affleck, in Fields, Strings and Critical Phenomena, Les Houches, Session XLIX, ed. by E. Brezin and J. ZinnJustin (North-Holland, Amsterdam, 1988).

[4] Precisely speaking, "unit cell" in this letter refers to the primitive unit cell, namely the minimum unit of the periodic structure.

[5] I. Affleck, T. Kennedy, E. H. Lieb and H. Tasaki, Phys. Rev. Lett. 59, 899 (1987); Commun. Math. Phys. 115, 477 (1988); T. Kennedy, E. H. Lieb and H. Tasaki, J. Stat. Phys. 53383 (1988).

[6] K. Hida, J. Phys. Soc. Jpn. 63, 2359 (1994).

[7] M. Oshikawa, M. Yamanaka and I. Affleck, Phys. Rev. Lett. 78, 1984 (1997).

[8] M. Yamanaka, M. Oshikawa and I. Affleck, Phys. Rev. Lett. 79, 1110 (1997).

[9] E. H. Lieb, T. Schultz, and D. J. Mattis, Ann. Phys. (N.Y.) 16, 407 (1961).

[10] I. Affleck, Phys. Rev. B 37, 5186 (1988).

[11] R. B. Laughlin, Phys. Rev. B 23, 5362 (1981).

[12] R. Tao and Y.-S. Wu, Phys. Rev. B 30, 1097 (1984).

[13] I. Affleck and E. H. Lieb, Lett. Math. Phys. 12, 57 (1986).

[14] While the LSM argument needs the assumption of shortrange particle hopping, there is apparently no such restriction in the present argument. However, an implicit assumption in Ref. 11 and in the present argument is that the finite gap does not collapse by the AB effect. This may be violated if very-long-range hoppings are introduced.

[15] D. H. Lee and R. Shankar, Phys. Rev. Lett. 65, 1490 (1990).

[16] F. D. M. Haldane, Phys. Rev. Lett. 61, 1029 (1988).

[17] D. S. Rokhsar and S. A. Kivelson, Phys. Rev. Lett. 61, 2376 (1988); N. Read and B. Chakraborty, Phys. Rev. B 40, 7133 (1989).

[18] S. Sachdev and N. Read, Int. J. Mod. Phys. B 5, 219 (1991); S. Sachdev, Phys. Rev. B 45, 12377 (1992); S. Sachdev and M. Vojta, cond-mat/9910231.

[19] G. Misguich, C. Lhullier, B. Bernu and C. Waldtmann, Phys. Rev. B 60, 1064 (1999).

[20] H. Nishimori and S. Miyashita, J. Phys. Soc. Jpn. 55, 4448 (1986); A. Kolezhuk, Phys. Rev. B 59, 4181 (1999); H. Kageyama et al., Phys. Rev. Lett. 82, 3168 (1999); T. Momoi, H. Sakamoto and K. Kubo, Phys. Rev. B 59, 9491 (1999); T. Momoi and K. Totsuka, condmat/9910057; Y. Fukumoto and A. Oguchi, preprint.

[21] P. Azaria et al., Phys. Rev. Lett. 81, 1694 (1998); S. K. Pati and R. R. P. Singh, cond-mat/9903434.

[22] H. Frahm and C. Sobiella, cond-mat/9907258.

[23] P. Lecheminant et al., Phys. Rev. B 56, 2521 (1997). 